\title{
Survival Mechanisms Used by Some Leishmania Species to Escape Neutrophil Killing
}

\author{
Ivo B. Regli, Katiuska Passelli, Benjamin P. Hurrell and Fabienne Tacchini-Cottier*
}

Department of Biochemistry, WHO Immunology Research and Training Collaborative Center, University of Lausanne, Lausanne, Switzerland

\section{OPEN ACCESS}

Edited by: Celio Geraldo Freire De Lima Universidade Federal do Rio de Janeiro, Brazil

Reviewed by:

Hira Nakhasi,

Center for Biologics Evaluation and Research (FDA), United States Marise Pinheiro Nunes,

Fundação Oswaldo Cruz

(Fiocruz), Brazil

*Correspondence:

Fabienne Tacchini-Cottier fabienne.tacchini-cottier@unil.ch

Summary sentence: In this mini-review, we discuss the dual function of neutrophils that may efficiently kill or in contrast, serve as a safe transient shelter for Leishmania spp. allowing in some cases their replication.

Specialty section: This article was submitted to Microbial Immunology, a section of the journal

Frontiers in Immunology

Received: 10 October 2017 Accepted: 31 October 2017 Published: 16 November 2017

Citation:

Regli IB, Passelli K, Hurrell BP and Tacchini-Cottier F (2017) Survival

Mechanisms Used by Some Leishmania Species to Escape Neutrophil Killing.

Front. Immunol. 8:1558. doi: 10.3389/fimmu.2017.01558
Neutrophils are the most abundant leukocytes in human blood. Upon microbial infection, they are massively and rapidly recruited from the circulation to sites of infection where they efficiently kill pathogens. To this end, neutrophils possess a variety of weapons that can be mobilized and become effective within hours following infection. However, several microbes including some Leishmania spp. have evolved a variety of mechanisms to escape neutrophil killing using these cells as a basis to better invade the host. In addition, neutrophils are also present in unhealing cutaneous lesions where their role remains to be defined. Here, we will review recent progress in the field and discuss the different strategies applied by some Leishmania parasites to escape from being killed by neutrophils and as recently described for Leishmania mexicana, even replicate within these cells. Subversion of neutrophil killing functions by Leishmania is a strategy that allows parasite spreading in the host with a consequent deleterious impact, transforming the primary protective role of neutrophils into a deleterious one.

Keywords: Leishmania, neutrophils, Leishmania survival, neutrophil extracellular traps, reactive oxygen species, neutrophil granules, Leishmania replication

\section{NEUTROPHILS AND Leishmania: A MULTIFACETED STORY}

Neglected parasitic diseases are affecting more than one million people worldwide. Amongst them, leishmaniases are a complex of diseases that affects 2 million people per year across 98 countries. The Leishmania protozoan parasites are transmitted by blood-sucking sand flies that deposit the parasites in the mammalian skin during their blood meal. There are more than 20 different Leishmania species worldwide. The infecting species together with host factors determine the various clinical manifestations leishmaniasis can have as well as the outcome of the disease. Cutaneous leishmaniasis is the most predominant form of the diseases. Following infection, an ulcerative lesion usually appears near the insect bite site. In mucocutaneous leishmaniasis, the disease affects the mucocuatenous tissues of the oro-naso-pharyngeal areas and often leads to local tissue destruction and death due to secondary infections if left untreated. Visceral leishmaniasis is characterized by hepatosplenomegaly and impeded bone marrow function due to the proliferation of parasites in macrophages within these organs. If not treated, visceral leishmaniasis patients develop cachexia, pancytopenia, subsequent immunosuppression and they eventually succumb to their disease (1). There are several treatments available against leishmaniasis of which pentavalent antimonials have been the standard of care for decades. However, these drugs have many adverse effects and the emergence of drug-resistant parasites is increasing worldwide. As the increase in drug resistance renders the available therapeutics less efficient, the need of efficient vaccines and a better understanding of the diseases is crucial to fight leishmaniases $(2,3)$. 
Neutrophils are massively and rapidly recruited to sites of injury and microbial infections. They are the most abundant leukocytes in human blood. Neutrophils play very important roles in innate immunity and in the regulation of adaptive immune response $(4,5)$. They are well known for their antimicrobial functions, playing a decisive role in innate host defense against a variety of pathogens, including bacteria and fungi. To kill microbes, neutrophils possess an arsenal of weapons that include phagocytosis and subsequent microbe degradation within phagolysosomes, where granules fuse to rapidly release their microbicidal agents. Neutrophils can degranulate their granule content also in the local microenvironment and they can also kill pathogens through the production of reactive oxygen species (ROS). In addition, neutrophils can extrude neutrophil extracellular traps (NETs) that consist of a DNA backbone associated with microbicidal proteins. NETs allow entrapping of the pathogens, preventing their spread, and in some cases killing them (6). Cytokines and chemokines released by neutrophils are involved in the activation and/or recruitment of other innate cells thereby contributing to the shaping and development of an adaptive immune response $(7,8)$. The relevance of the role played by neutrophils in the fight against many infections is underlined by the susceptibility to repeated life-threatening bacterial and fungal infections observed in patients suffering from genetically inherited or acquired neutropenia or who have neutrophils with functional defects (9). The important role of neutrophils in regulating defense against parasites and some viruses has more recently emerged $(10,11)$ and increasing evidence points out to a crucial role for neutrophils in leishmaniasis disease outcome (10-13).

In contrast to their well-described protective roles in many infections, neutrophils may play a detrimental role in leishmaniasis disease development, at least in some instances. In addition to their early recruitment following infection, neutrophils were reported to infiltrate damaged tissues of human mucosal leishmaniasis (14) and to be present in the chronic form of the disease in human and animals (14-20). Following experimental infection with most Leishmania spp. neutrophils are rapidly and massively recruited to the site of parasite inoculation where they rapidly phagocytose most of the parasites present. Several groups have used genetically neutropenic mice or mice rendered neutropenic by injection of anti-neutrophil antibodies to show the importance of this early wave of neutrophil on disease outcome. Collectively, most of these studies reported that neutropenic mice had a better disease outcome, indicating a negative role for neutrophils in some forms of cutaneous leishmaniasis (2, 21-24). In contrast, neutrophils may facilitate parasite clearance as observed for Leishmania braziliensis and Leishmania amazonensis (25-30) and for Leishmania donovani (31). However, L. amazonensis killing appeared to be parasite stage-dependent as promastigotes, the infecting form of the parasites, but not amastigotes, the intracellular replicative forms of the parasite, were killed in vitro by neutrophils (32).

One of the immune evasion strategies used by Leishmania parasites may be linked to the status of neutrophil apoptosis as phagocytosis of apoptotic neutrophils has been shown to impair dendritic cells (DCs) maturation and the development of an efficient adaptive immune response [reviewed in Ref. (7)]. Indeed, internalization of apoptotic Leishmania major-infected neutrophils by DCs impaired development of Leishmania-specific immune response $(33,34)$. Interaction of apoptotic neutrophils with macrophages also has a negative impact on the disease (35). Following Leishmania delivery by sand fly bite or needle inoculation, parasites were reported to induce, delay or have no impact on neutrophil apoptosis, depending on the Leishmania spp. or the origin of neutrophils. Leishmania mexicana did not influence dermal neutrophil survival ex vivo (36) and L. infantum did not induce neutrophil apoptosis in vitro (37). In contrast, L. brasiliensis induced neutrophil apoptosis, at least in vitro (30). L. major infection induced murine neutrophil apoptosis in the dermis $(22,34)$ while it delayed human blood-derived neutrophil apoptosis $(22,34,38,39)$. These results suggest that the effect of Leishmania on neutrophil apoptosis may differ between murine and human neutrophils, or the difference observed may come from the diverse Leishmania spp. or neutrophil origins.

Recent data reported that a subset of low density neutrophils expressing HLA-DR express high levels of PDL1 in human CL and VL patients $(19,40)$, a marker promoting T cell exhaustion. These data suggest a novel negative role for this neutrophil subset in leishmaniasis.

\section{THE DISTINCT MECHANISMS USED BY Leishmania spp. TO ESCAPE KILLING BY NEUTROPHILS}

Leishmania are using neutrophils transiently to finally be ingested by macrophages, their final host. The parasites may be released by dying neutrophils and/or infected apoptotic neutrophils may be phagocytosed by macrophages. This latter process referred to as the "Trojan horse" entry in macrophages, confers a silent entry for the parasites in these cells (41). We will now discuss the several mechanisms used by some Leishmania spp. to escape neutrophil killing and even in some cases how the parasites can use these cells to replicate, collectively resulting in a negative impact on disease outcome.

Using in vivo two-photon imaging, intact and live parasites have been detected in neutrophils during the first days of L. major and L. mexicana infections, revealing that a good proportion of parasites can resist neutrophil microbicidal functions $(22,36)$. Several strategies used by Leishmania parasites to escape killing by neutrophils have been described. During neutrophil development there is a continuity of granule formation, including azurophil granules (primary or peroxidasepositive granules), specific (secondary granules), and gelatinase granules (tertiary granules). Secretory granules are formed last (42). During the maturation of myeloblasts into neutrophils, more than 300 different proteins are stored into granules. One of the ways parasites may survive in neutrophils is through interference in the process of granule fusion with the Leishmania containing phagosome. In vitro studies showed that L. major and $L$. donovani promastigotes regulate granule fusion with phagosomes, allowing azurophil but preventing specific and 
gelatinase granule fusion with parasite-containing phagosomes (43). This prevents their destruction by neutrophils microbicidal granule contents (Figure 1A). In addition, L. donovani was shown to traffic to non-lytic compartments within neutrophils (44), establishing yet another strategy to escape the neutrophil killing machinery (Figure 1B).

In addition to the release of antimicrobial molecules, the assembly of a functional NADPH oxidase (NOX2) is playing a crucial role for neutrophil microbicidal function (45). NOX2 assembly is inducing the generation of reactive oxygen species (ROS), a process called oxidative burst. Interference with oxidative burst increases pathogen survival within neutrophils. It has been shown that L. major does not elicit the generation of ROS upon phagocytosis by human neutrophils (Figure 1C)
(43). However, L. braziliensis induce high levels of ROS production upon infection of human and murine neutrophils but ROS generation in human neutrophils did not affect parasite survival $(31,45)$. In addition to its major role in neutrophil intracellular killing functions, NOX2-mediated generation of ROS has also been reported to be crucial for classical (NADPH-dependent) NET formation. This is exemplified by the lack of NET formation in patients with chronic granulomatous disease $(46,47)$ and restoration of NET formation in these patients upon reintroduction of NOX2 by genetic engineering (48). Moreover, there also exists ROS-independent NET release. L. amazonensis promastigotes were shown to elicit both types of NETs and be killed by them (29). Thus, the impact of parasites on ROS formation is also Leishmania spp. dependent.

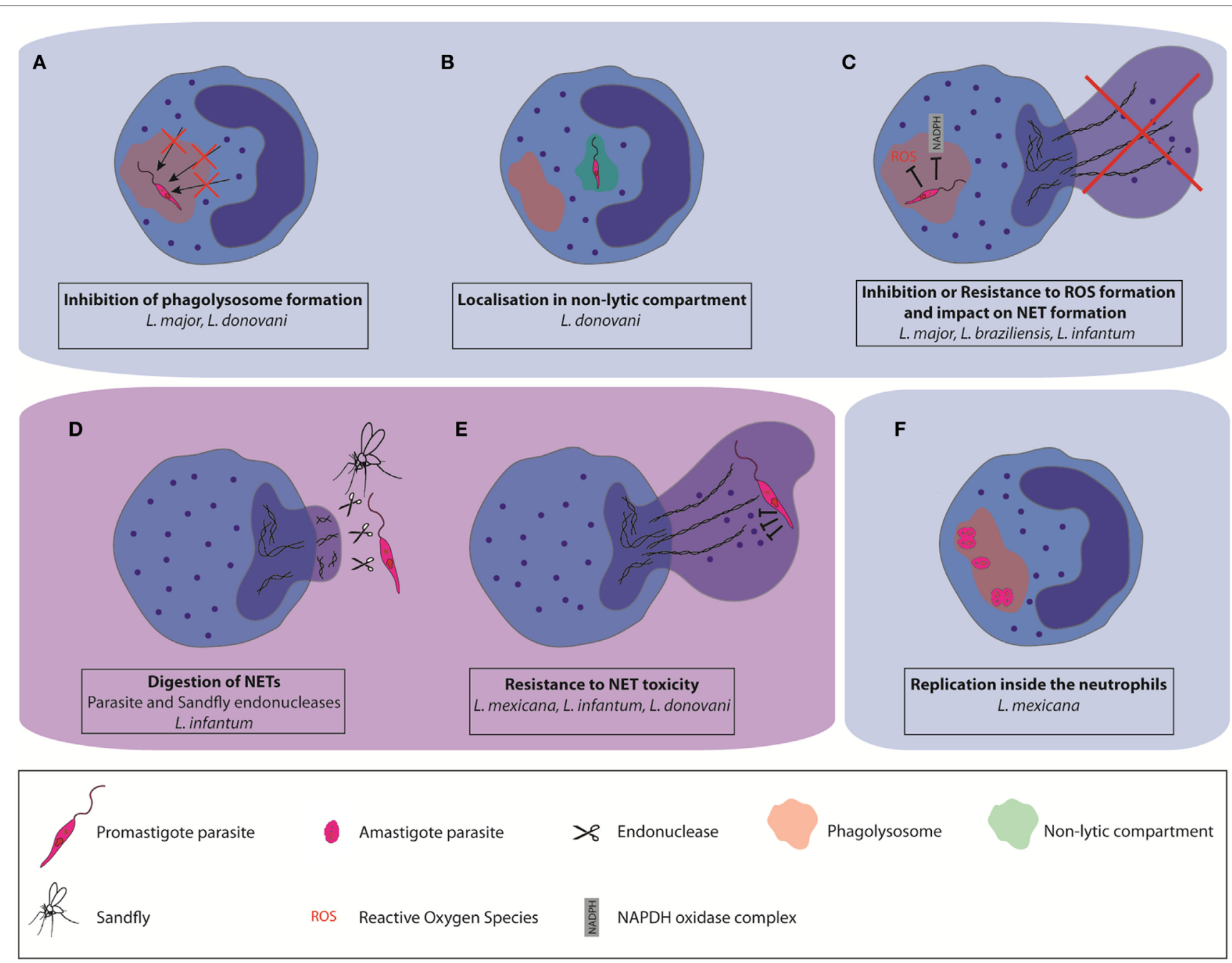

FIGURE 1 | Different mechanisms used by some Leishmania spp. to escape neutrophil killing. Leishmania can impair parasite destruction by neutrophils (A) by affecting the formation of mature phagolysosomes and their fusion with neutrophil granules, (B) by localization in non-lytic compartments, and (C) by resisting to the toxicity associated with reactive oxygen species production. Some Leishmania spp. can also resist to the microbicity associated with neutrophil extracellular trap (NET) formation (D) by directly inhibiting NET formation, or by digestion of the NET scaffold using pathogen-or vector-derived endonucleases (E). They can also resist NET antimicrobial factors through the expression of protease-resistant surface molecules. (F) A subset of $L$. mexicana amastigotes was shown to replicate in neutrophils. 


\section{PATHOGENS ESCAPE FROM NETS}

Upon activation, neutrophils can form NETs that can entrap and often kill pathogens, reviewed in Ref. (49). However, several microbes including some Leishmania spp. have developed various mechanisms to escape NET trapping and/or killing. Whether parasites are killed or not by NETs depends on the involved Leishmania spp. For instance, in humans, L. amazonensis was shown to induce NET formation and to be killed by them (50). In contrast, NETs failed to kill (36) L. infantum (51) and L. donovani (52) parasites. Furthermore, murine NETs were not able to kill L. mexicana (36).

A very efficient strategy used by Leishmania infantum (51) is to prevent NET formation by suppressing or inducing decreased efficiency of the oxidative burst (Figure 1C).

As another strategy to avoid NET killing, several microbes express nucleases that degrade the NET DNA backbone. For example, surface DNAse and wall anchored nuclease expression were reported in Gram-positive bacteria (53-56) and for several Gram-negative bacteria $(53,57-59)$. NET degrading endonucleases have also been reported in Gram-negative bacteria (60-62). Expression of the enzyme 3'nucleotidase/nuclease by Leishmania also contributes to protection from the microbicidal activity of NETs as shown for L. infantum (51). In addition, the parasite sand fly vector may interfere with NET formation. The saliva of the New World Leishmania vector, Lutzomyia longipalpis, was shown to contain an endonuclease capable of degrading NETs (63). As salivary gland proteins are deposited by the sandfly in the host during the insect blood meal its endonucleases may indirectly influence the role of NETs in the disease pathogenesis (Figure 1D).

Microbes may also avoid NET killing through the synthesis of cell surface components rendering them resistant to NETassociated protease activity (Figure 1E). This has been observed for L. amazonensis and L. donovani. Leishmania surface coat is densely packed with lipophosphoglycan (LPG), a glycoconjugate that is polymorphic among Leishmania spp. and which is differentially expressed in the infective promastigote form compared to the replicative amastigote form (64). In L. amazonensis, LPG was shown to induce NET formation, and confer resistance to NET-mediated killing by forming a thick glycocalyx that protects the parasite from microbicidal agents (50). In contrast, LPG of $L$. donovani, was shown not to induce NET formation, although it also conferred protection against NET mediated parasite killing (52). Peripheral blood neutrophils from active VL patients were unable to release NETs despite an active phenotype (65), showing that the replicating amastigote stage of the parasites also has an impact on neutrophil functions, contributing to the pathology of the disease.

\section{NEUTROPHILS AS A PLACE TO REPLICATE}

Neutrophils are short-lived non-dividing cells that become rapidly apoptotic in the circulation. However, during inflammation and infection, the neutrophil lifespan can be extended to several days (66), although it still remains difficult to estimate neutrophil lifespan in tissues, mostly due to technical issues. For some Leishmania spp. transient inhibition or delay of neutrophil apoptosis is an obvious strategy to allow prolongation of their presence within these cells. The PI3K/AKT, ERK1/2 p28MAPK pathways which maintain expression of the antiapoptotic Mcll protein were shown to contribute to prolonged neutrophil lifespan in L. major infection (67).

The induction of delayed neutrophil apoptosis together with the inhibition of neutrophil killing machinery elicited by some Leishmania spp. suggested that the parasite could use these cells to replicate. Leishmania parasites have two life cycle stages, the infective flagellated promastigote form which is elongated with a size comprised between 6 and $12 \mu \mathrm{m}$, not including the flagellum length, and the replicative, non-flagellated amastigote form, which is intracellular and of smaller size $(3-5 \mu \mathrm{m})$. The sand fly is depositing in the skin metacyclic promastigotes, a process inducing rapid recruitment of neutrophils. It is therefore not surprising that most studies investigating interactions between neutrophils and Leishmania have been performed with the promastigote form of the parasite, reviewed in Ref. $(12,13,24)$. In addition, neutrophils have been detected in smears of unhealing cutaneous lesions of $L$. braziliensis patients, at a time when the parasite is in its intracellular amastigote form. The presence of neutrophil-attracting chemokine mRNA was observed in biopsies of patients with chronic lesions due to L. panamensis and $L$. braziliensis, suggesting neutrophil presence in the lesion. Also, neutrophils were observed in biopsies of tegumentary leishmaniasis patients $(14,68-71)$. Furthermore, neutrophil presence was also observed in unhealing lesions of experimental cutaneous leishmaniasis following L. major $(18,21)$ and L. mexicana infection (36). Very few studies have investigated the interactions between neutrophils and the amastigote form of the parasite. The group of Soong was the first to show that neutrophils internalized in vitro $L$. amazonensis and $L$. braziliensis amastigotes. While L. amazonensis amastigotes survived in neutrophils, L. braziliensis amastigotes were efficiently killed $(28,32)$. We recently reported that L. mexicana amastigotes are also internalized and survive in neutrophils in vitro. After overnight incubation, we observed an average of one amastigote per neutrophils. In contrast, the majority of lesion-derived neutrophils harbored $>2$ intact amastigotes per neutrophil. Imaging of the lesionderived neutrophils showed the presence of several aligned amastigotes within neutrophils, suggesting possible parasite replication in these cells. Parasite uptake by neutrophils was relatively neutral, eliciting low level of apoptosis or neutrophil activation in infected neutrophils (20). To measure parasite replication, we generated transgenic parasites expressing a photoconvertible GFP mKikume gene (72). These $L$. mex $^{\text {SWITCH }}$ parasites express constitutively green fluorescence that can be converted to red fluorescence upon exposure to a pulse of violet light. Upon cell division, the photoconverted red proteins are diluted as de novo green protein in synthesized, and the fluorescence recovery after conversion (FRAC) is measured in dividing cells. Analysis of FRAC by imaging flow cytometry and time-lapse microscopy revealed that, $48 \mathrm{~h}$ after photoconversion, a subset of highly infected neutrophils containing more than 4 amastigotes per cell 
showed high replication (Figure 1F). Amastigotes were found in large vesicular acidic compartment. In macrophages, Leishmania amastigotes reside in phagolysosome-like compartments called parasitophorous vacuoles (PVs) where they multiply. For most Leishmania spp. one amastigote is enclosed within these PVs with little vacuole space. However, L. mexicana and L. amazonensis form upon division communal large PVs containing numerous amastigotes, a process diluting toxic components and directly linked to parasite evasion to host immune responses $(73,74)$. We observed larger Lysosensor-positive vacuoles in L. mexicana infected neutrophils (20), suggesting the formation of communal PVs in neutrophils. It remains to be determined whether the replication of amastigotes in neutrophils is linked to the presence of these large PVs.

The majority of parasite replication is taking place in macrophages, and most lesional parasites divide at a slow rate even if, as observed in vitro, there is likely variability in the growth rates of parasites in unhealing cutaneous lesions (75). Indeed, in a recent study a small subset of parasites that appeared to divide rapidly was reported. These parasites could use neutrophils as a safe transient place to replicate.

The demonstration that a subset of L. mexicana parasites is able to replicate within neutrophils revealed a novel role of neutrophils that can act as a niche for parasite replication during the chronic phase of infection. However, there very likely exist differences in the ability of the invading Leishmania spp. to replicate in neutrophils. These could originate from parasite factors but also from host factors.

\section{CONCLUDING REMARKS}

The primary function of neutrophils in innate immunity resides in killing invading microorganisms. It is therefore not surprising that some pathogens have evolved several ways to escape elimination by these cells, allowing their silent entry in the host and even sometimes their replication in these cells. Caution in the interpretation of some of these studies should be taken as most human studies are performed with peripheral blood neutrophils that functionally differ from extravasated neutrophils present in inflamed tissues. To better understand the relevance

\section{REFERENCES}

1. Herwaldt BL. Leishmaniasis. Lancet (1999) 354(9185):1191-9. doi:10.1016/ S0140-6736(98)10178-2

2. Kaye P, Scott P. Leishmaniasis: complexity at the host-pathogen interface. Nat Rev Microbiol (2011) 9(8):604-15. doi:10.1038/nrmicro2608

3. McGwire BS, Satoskar AR. Leishmaniasis: clinical syndromes and treatment. QJM (2014) 107(1):7-14. doi:10.1093/qjmed/hct116

4. Mocsai A. Diverse novel functions of neutrophils in immunity, inflammation, and beyond. J Exp Med (2013) 210(7):1283-99. doi:10.1084/jem.20122220

5. Nauseef WM, Borregaard N. Neutrophils at work. Nat Immunol (2014) 15(7):602-11. doi:10.1038/ni.2921

6. Brinkmann V, Reichard U, Goosmann C, Fauler B, Uhlemann Y, Weiss DS, et al. Neutrophil extracellular traps kill bacteria. Science (2004) 303(5663): 1532-5. doi:10.1126/science. 1092385

7. Schuster S, Hurrell B, Tacchini-Cottier F. Crosstalk between neutrophils and dendritic cells: a context-dependent process. JLeukoc Biol (2013) 94(4):671-5. doi:10.1189/jlb.1012540 of neutrophil functions in vivo, experimental murine models are used. However, it should be kept in mind that functional differences exist between mouse and human neutrophils as well, including differences in the antimicrobial repertoire and number of circulating neutrophils (76). That being said, the generation of new tools such as two-photon microscopy imaging (77) and the use of photo-switchable pathogens (78) for probing pathogen biology during infections should allow finer investigation of the mechanisms used by pathogens to promote their own survival in neutrophils in vivo. Furthermore, neutrophils appear to be a more heterogeneous cell population than previously anticipated (79) and new markers defining mature from immature circulating neutrophils are emerging (80). It will thus be interesting to assess whether selective Leishmania spp. transient survival and/or replication occur in a specific neutrophil subset, while Leishmania killing would take place in other subsets.

Survival of pathogens in neutrophils is not specific to Leishmania, indeed several bacteria, fungi or viruses are also able to escape neutrophil killing and use these cells to propagate in the host, reviewed in Ref. (81). For instance, intracellular bacteria including Francisella tularensis (82), Neisseira gonorrhoae (83), Chlamydia pneumonia (84); and more recently, Yersina spp. (85) have been shown to replicate in vitro in human or murine neutrophils, suggesting that not only Leishmania parasites but also other pathogens are diverting the primary neutrophil killing function to their own benefit and dissemination in the invaded host. Finer understanding of the mechanisms used by some Leishmania spp. to block neutrophil effector functions will be important in the design of prophylactic or therapeutic measures taken against leishmaniasis.

\section{AUTHOR CONTRIBUTIONS}

IR and FTC wrote the review. BH, KP, and IR contributed to the figures. All authors provided input to the review.

\section{FUNDING}

We acknowledge the financial support of the Swiss National Foundation for Scientific Research (310030_166651/1 to FC).

8. Scapini P, Cassatella MA. Social networking of human neutrophils within the immune system. Blood (2014) 124(5):710-9. doi:10.1182/blood-201403-453217

9. Klein C. Genetic defects in severe congenital neutropenia: emerging insights into life and death of human neutrophil granulocytes. Annu Rev Immunol (2011) 29:399-413. doi:10.1146/annurev-immunol-030409-101259

10. Kolaczkowska E, Kubes P. Neutrophil recruitment and function in health and inflammation. Nat Rev Immunol (2013) 13(3):159-75. doi:10.1038/nri3399

11. Drescher B, Bai F. Neutrophil in viral infections, friend or foe? Virus Res (2013) 171(1):1-7. doi:10.1016/j.virusres.2012.11.002

12. Carlsen ED, Liang Y, Shelite TR, Walker DH, Melby PC, Soong L. Permissive and protective roles for neutrophils in leishmaniasis. Clin Exp Immunol (2015) 182(2):109-18. doi:10.1111/cei.12674

13. Hurrell BP, Regli IB, Tacchini-Cottier F. Different Leishmania species drive distinct neutrophil functions. Trends Parasitol (2016) 32(5):392-401. doi:10.1016/ j.pt.2016.02.003

14. Boaventura VS, Santos CS, Cardoso CR, de Andrade J, Dos Santos WL, Clarencio J, et al. Human mucosal leishmaniasis: neutrophils infiltrate areas 
of tissue damage that express high levels of Th17-related cytokines. Eur J Immunol (2010) 40(10):2830-6. doi:10.1002/eji.200940115

15. Morgado FN, Schubach A, Rosalino CM, Quintella LP, Santos G, Salgueiro M, et al. Is the in situ inflammatory reaction an important tool to understand the cellular immune response in American tegumentary leishmaniasis? Br J Dermatol (2008) 158(1):50-8. doi:10.1111/j.1365-2133.2007. 08255. $\mathrm{x}$

16. Vercosa BL, Melo MN, Puerto HL, Mendonca IL, Vasconcelos AC. Apoptosis, inflammatory response and parasite load in skin of Leishmania (Leishmania) chagasi naturally infected dogs: a histomorphometric analysis. Vet Parasitol (2012) 189(2-4):162-70. doi:10.1016/j.vetpar.2012.04.035

17. Dantas ML, Oliveira JM, Carvalho L, Passos ST, Queiroz A, Guimaraes LH, et al. Comparative analysis of the tissue inflammatory response in human cutaneous and disseminated leishmaniasis. Mem Inst Oswaldo Cruz (2014) 109(2):202-9. doi:10.1590/0074-0276130312

18. Charmoy M, Hurrell BP, Romano A, Lee SH, Ribeiro-Gomes F, Riteau N, et al. The Nlrp3 inflammasome, IL-1beta, and neutrophil recruitment are required for susceptibility to a nonhealing strain of Leishmania major in C57BL/6 mice. Eur J Immunol (2016) 46(4):897-911. doi:10.1002/eji. 201546015

19. Davis RE, Sharma S, Conceicao J, Carneiro P, Novais F, Scott P, et al. Phenotypic and functional characteristics of HLA-DR+ neutrophils in Brazilians with cutaneous leishmaniasis. J Leukoc Biol (2017) 101(3):739-49. doi:10.1189/jlb.4A0915-442RR

20. Hurrell BP, Beaumann M, Heyde S, Regli IB, Muller AJ, Tacchini-Cottier F. Frontline science: Leishmania mexicana amastigotes can replicate within neutrophils. J Leukoc Biol (2017). doi:10.1189/jlb.4HI0417-158R

21. Tacchini-Cottier F, Zweifel C, Belkaid Y, Mukankundiye C, Vasei M, Launois $\mathrm{P}$, et al. An immunomodulatory function for neutrophils during the induction of a CD4+ Th2 response in BALB/c mice infected with Leishmania major. J Immunol (2000) 165(5):2628-36. doi:10.4049/jimmunol. 165.5.2628

22. Peters NC, Egen JG, Secundino N, Debrabant A, Kimblin N, Kamhawi S, et al. In vivo imaging reveals an essential role for neutrophils in leishmaniasis transmitted by sand flies. Science (2008) 321(5891):970-4. doi:10.1126/ science.1159194

23. Charmoy M, Auderset F, Allenbach C, Tacchini-Cottier F. The prominent role of neutrophils during the initial phase of infection by Leishmania parasites. J Biomed Biotechnol (2010) 2010:719361. doi:10.1155/2010/719361

24. Ribeiro-Gomes FL, Sacks D. The influence of early neutrophil-Leishmania interactions on the host immune response to infection. Front Cell Infect Microbiol (2012) 2:59. doi:10.3389/fcimb.2012.00059

25. Souza-Lemos C, de-Campos SN, Teva A, Corte-Real S, Fonseca EC, Porrozzi R, et al. Dynamics of immune granuloma formation in a Leishmania braziliensis-induced self-limiting cutaneous infection in the primate Macaca mulatta. J Pathol (2008) 216(3):375-86. doi:10.1002/path.2403

26. Novais FO, Santiago RC, Bafica A, Khouri R, Afonso L, Borges VM, et al. Neutrophils and macrophages cooperate in host resistance against Leishmania braziliensis infection. J Immunol (2009) 183(12):8088-98. doi:10.4049/ jimmunol.0803720

27. Tavares NM, Araujo-Santos T, Afonso L, Nogueira PM, Lopes UG, Soares RP, et al. Understanding the mechanisms controlling Leishmania amazonensis infection in vitro: the role of LTB4 derived from human neutrophils. J Infect Dis (2014) 210(4):656-66. doi:10.1093/infdis/jiu158

28. Carlsen ED, Jie Z, Liang Y, Henard CA, Hay C, Sun J, et al. Interactions between neutrophils and Leishmania braziliensis amastigotes facilitate cell activation and parasite clearance. J Innate Immun (2015) 7(4):354-63. doi:10.1159/ 000373923

29. Rochael NC, Guimaraes-Costa AB, Nascimento MT, DeSouza-Vieira TS, Oliveira MP, Garcia e Souza LF, et al. Classical ROS-dependent and early/ rapid ROS-independent release of neutrophil extracellular traps triggered by Leishmania parasites. Sci Rep (2015) 5:18302. doi:10.1038/srep18302

30. Falcao SA, Weinkopff T, Hurrell BP, Celes FS, Curvelo RP, Prates DB, et al. Exposure to Leishmania braziliensis triggers neutrophil activation and apoptosis. PLoS Negl Trop Dis (2015) 9(3):e0003601. doi:10.1371/journal.pntd. 0003601

31. McFarlane E, Perez C, Charmoy M, Allenbach C, Carter KC, Alexander J, et al. Neutrophils contribute to development of a protective immune response during onset of infection with Leishmania donovani. Infect Immun (2008) 76(2):532-41. doi:10.1128/IAI.01388-07

32. Carlsen ED, Hay C, Henard CA, Popov V, Garg NJ, Soong L. Leishmania amazonensis amastigotes trigger neutrophil activation but resist neutrophil microbicidal mechanisms. Infect Immun (2013) 81(11):3966-74. doi:10.1128/IAI.00770-13

33. Ribeiro-Gomes FL, Peters NC, Debrabant A, Sacks DL. Efficient capture of infected neutrophils by dendritic cells in the skin inhibits the early anti-Leishmania response. PLoS Pathog (2012) 8(2):e1002536. doi:10.1371/ journal.ppat.1002536

34. Ribeiro-Gomes FL, Romano A, Lee S, Roffe E, Peters NC, Debrabant A et al. Apoptotic cell clearance of Leishmania major-infected neutrophils by dendritic cells inhibits $\mathrm{CD} 8(+)$ T-cell priming in vitro by Mer tyrosine kinase-dependent signaling. Cell Death Dis (2015) 6:e2018. doi:10.1038/ cddis.2015.351

35. Afonso L, Borges VM, Cruz H, Ribeiro-Gomes FL, DosReis GA, Dutra AN, et al. Interactions with apoptotic but not with necrotic neutrophils increase parasite burden in human macrophages infected with Leishmania amazonensis. J Leukoc Biol (2008) 84(2):389-96. doi:10.1189/jlb.0108018

36. Hurrell BP, Schuster S, Grun E, Coutaz M, Williams RA, Held W, et al. Rapid sequestration of Leishmania mexicana by neutrophils contributes to the development of chronic lesion. PLoS Pathog (2015) 11(5):e1004929. doi:10.1371/journal.ppat.1004929

37. Marques CS, Passero LF, Vale-Gato I, Rodrigues A, Rodrigues OR, Martins C, et al. New insights into neutrophil and Leishmania infantum in vitro immune interactions. Comp Immunol Microbiol Infect Dis (2015) 40:19-29. doi:10.1016/j.cimid.2015.03.003

38. Aga E, Katschinski DM, van Zandbergen G, Laufs H, Hansen B, Muller K, et al. Inhibition of the spontaneous apoptosis of neutrophil granulocytes by the intracellular parasite Leishmania major. J Immunol (2002) 169(2):898-905. doi:10.4049/jimmunol.169.2.898

39. Sarkar A, Aga E, Bussmeyer U, Bhattacharyya A, Moller S, Hellberg L, et al. Infection of neutrophil granulocytes with Leishmania major activates ERK $1 / 2$ and modulates multiple apoptotic pathways to inhibit apoptosis. Med Microbiol Immunol (2013) 202(1):25-35. doi:10.1007/s00430-0120246-1

40. Sharma S, Davis RE, Srivastva S, Nylen S, Sundar S, Wilson ME. A subset of neutrophils expressing markers of antigen-presenting cells in human visceral leishmaniasis. J Infect Dis (2016) 214(10):1531-8. doi:10.1093/infdis/ jiw394

41. van Zandbergen G, Klinger M, Mueller A, Dannenberg S, Gebert A, Solbach W, et al. Cutting edge: neutrophil granulocyte serves as a vector for Leishmania entry into macrophages. J Immunol (2004) 173(11):6521-5. doi:10.4049/jimmunol.173.11.6521

42. Borregaard N, Sorensen OE, Theilgaard-Monch K. Neutrophil granules: a library of innate immunity proteins. Trends Immunol (2007) 28(8):340-5. doi:10.1016/j.it.2007.06.002

43. Mollinedo F, Janssen H, de la Iglesia-Vicente J, Villa-Pulgarin JA, Calafat J. Selective fusion of azurophilic granules with Leishmania-containing phagosomes in human neutrophils. J Biol Chem (2010) 285(45):34528-36. doi:10.1074/jbc.M110.125302

44. Gueirard P, Laplante A, Rondeau C, Milon G, Desjardins M. Trafficking of Leishmania donovani promastigotes in non-lytic compartments in neutrophils enables the subsequent transfer of parasites to macrophages. Cell Microbiol (2008) 10(1):100-11. doi:10.1111/j.1462-5822.2007.01018.x

45. Nauseef WM. Biological roles for the NOX family NADPH oxidases. J Biol Chem (2008) 283(25):16961-5. doi:10.1074/jbc.R700045200

46. Fuchs TA, Abed U, Goosmann C, Hurwitz R, Schulze I, Wahn V, et al. Novel cell death program leads to neutrophil extracellular traps. J Cell Biol (2007) 176(2):231-41. doi:10.1083/jcb.200606027

47. von Kockritz-Blickwede M, Goldmann O, Thulin P, Heinemann K, NorrbyTeglund A, Rohde $\mathrm{M}$, et al. Phagocytosis-independent antimicrobial activity of mast cells by means of extracellular trap formation. Blood (2008) 111(6):3070-80. doi:10.1182/blood-2007-07-104018

48. Bianchi M, Hakkim A, Brinkmann V, Siler U, Seger RA, Zychlinsky A, et al. Restoration of NET formation by gene therapy in CGD controls aspergillosis. Blood (2009) 114(13):2619-22. doi:10.1182/blood-2009-05221606 
49. Sorensen OE, Borregaard N. Neutrophil extracellular traps - the dark side of neutrophils. J Clin Invest (2016) 126(5):1612-20. doi:10.1172/jci84538

50. Guimaraes-Costa AB, Nascimento MT, Froment GS, Soares RP, Morgado FN, Conceicao-Silva F, et al. Leishmania amazonensis promastigotes induce and are killed by neutrophil extracellular traps. Proc Natl Acad Sci U S A (2009) 106(16):6748-53. doi:10.1073/pnas.0900226106

51. Guimaraes-Costa AB, DeSouza-Vieira TS, Paletta-Silva R, FreitasMesquita AL, Meyer-Fernandes JR, Saraiva EM. 3'-Nucleotidase/nuclease activity allows Leishmania parasites to escape killing by neutrophil extracellular traps. Infect Immun (2014) 82(4):1732-40. doi:10.1128/iai.01232-13

52. Gabriel C, McMaster WR, Girard D, Descoteaux A. Leishmania donovani promastigotes evade the antimicrobial activity of neutrophil extracellular traps. J Immunol (2010) 185(7):4319-27. doi:10.4049/jimmunol.1000893

53. Buchanan JT, Simpson AJ, Aziz RK, Liu GY, Kristian SA, Kotb M, et al. DNase expression allows the pathogen group A Streptococcus to escape killing in neutrophil extracellular traps. Curr Biol (2006) 16(4):396-400. doi:10.1016/j.cub.2005.12.039

54. Beiter K, Wartha F, Albiger B, Normark S, Zychlinsky A, HenriquesNormark B. An endonuclease allows Streptococcus pneumoniae to escape from neutrophil extracellular traps. Curr Biol (2006) 16(4):401-7. doi:10.1016/j. cub.2006.01.056

55. Morita C, Sumioka R, Nakata M, Okahashi N, Wada S, Yamashiro T, et al. Cell wall-anchored nuclease of Streptococcus sanguinis contributes to escape from neutrophil extracellular trap-mediated bacteriocidal activity. PLoS One (2014) 9(8):e103125. doi:10.1371/journal.pone.0103125

56. Berends ET, Horswill AR, Haste NM, Monestier M, Nizet V, von KockritzBlickwede M. Nuclease expression by Staphylococcus aureus facilitates escape from neutrophil extracellular traps. J Innate Immun (2010) 2(6):576-86. doi:10.1159/000319909

57. Sumby P, Barbian KD, Gardner DJ, Whitney AR, Welty DM, Long RD, et al. Extracellular deoxyribonuclease made by group A Streptococcus assists pathogenesis by enhancing evasion of the innate immune response. Proc Natl Acad Sci U S A (2005) 102(5):1679-84. doi:10.1073/pnas.0406641102

58. Derre-Bobillot A, Cortes-Perez NG, Yamamoto Y, Kharrat P, Couve E, Da Cunha V, et al. Nuclease A (Gbs0661), an extracellular nuclease of Streptococcus agalactiae, attacks the neutrophil extracellular traps and is needed for full virulence. Mol Microbiol (2013) 89(3):518-31. doi:10.1111/ mmi.12295

59. de Buhr N, Neumann A, Jerjomiceva N, von Kockritz-Blickwede M, Baums CG. Streptococcus suis DNase SsnA contributes to degradation of neutrophil extracellular traps (NETs) and evasion of NET-mediated antimicrobial activity. Microbiology (2014) 160(Pt 2):385-95. doi:10.1099/mic.0. 072199-0

60. Seper A, Hosseinzadeh A, Gorkiewicz G, Lichtenegger S, Roier S, Leitner DR, et al. Vibrio cholerae evades neutrophil extracellular traps by the activity of two extracellular nucleases. PLoS Pathog (2013) 9(9):e1003614. doi:10.1371/ journal.ppat.1003614

61. Mollerherm H, Neumann A, Schilcher K, Blodkamp S, Zeitouni NE, Dersch P, et al. Yersinia enterocolitica-mediated degradation of neutrophil extracellular traps (NETs). FEMS Microbiol Lett (2015) 362(23):fnv192. doi:10.1093/femsle/fnv192

62. Juneau RA, Stevens JS, Apicella MA, Criss AK. A thermonuclease of Neisseria gonorrhoeae enhances bacterial escape from killing by neutrophil extracellular traps. JInfect Dis (2015) 212(2):316-24. doi:10.1093/infdis/ jiv031

63. Chagas AC, Oliveira F, Debrabant A, Valenzuela JG, Ribeiro JM, Calvo E. Lundep, a sand fly salivary endonuclease increases Leishmania parasite survival in neutrophils and inhibits XIIa contact activation in human plasma. PLoS Pathog (2014) 10(2):e1003923. doi:10.1371/journal.ppat.1003923

64. Franco LH, Beverley SM, Zamboni DS. Innate immune activation and subversion of mammalian functions by Leishmania lipophosphoglycan. J Parasitol Res (2012) 2012:165126. doi:10.1155/2012/165126

65. Yizengaw E, Getahun M, Tajebe F, Cruz Cervera E, Adem E, Mesfin G, et al. Visceral leishmaniasis patients display altered composition and maturity of neutrophils as well as impaired neutrophil effector functions. Front Immunol (2016) 7:517. doi:10.3389/fimmu.2016.00517

66. Pillay J, den Braber I, Vrisekoop N, Kwast LM, de Boer RJ, Borghans JA, et al. In vivo labeling with $2 \mathrm{H}_{2} \mathrm{O}$ reveals a human neutrophil lifespan of 5.4 days. Blood (2010) 116(4):625-7. doi:10.1182/blood-2010-01-259028
67. Ruhland A, Leal N, Kima PE. Leishmania promastigotes activate PI3K/Akt signalling to confer host cell resistance to apoptosis. Cell Microbiol (2007) 9(1):84-96. doi:10.1111/j.1462-5822.2006.00769.x

68. Navas A, Vargas DA, Freudzon M, McMahon-Pratt D, Saravia NG, Gomez MA. Chronicity of dermal leishmaniasis caused by Leishmania panamensis is associated with parasite-mediated induction of chemokine gene expression. Infect Immun (2014) 82(7):2872-80. doi:10.1128/IAI.01133-13

69. Morgado FN, Nascimento MT, Saraiva EM, de Oliveira-Ribeiro C, Madeira Mde F, da Costa-Santos M, et al. Are neutrophil extracellular traps playing a role in the parasite control in active American tegumentary leishmaniasis lesions? PLoS One (2015) 10(7):e0133063. doi:10.1371/journal. pone. 0133063

70. Novais FO, Carvalho LP, Passos S, Roos DS, Carvalho EM, Scott P, et al. Genomic profiling of human Leishmania braziliensis lesions identifies transcriptional modules associated with cutaneous immunopathology. J Invest Dermatol (2015) 135(1):94-101. doi:10.1038/jid.2014.305

71. Conceicao J, Davis R, Carneiro PP, Giudice A, Muniz AC, Wilson ME, et al. Characterization of neutrophil function in human cutaneous leishmaniasis caused by Leishmania braziliensis. PLoS Negl Trop Dis (2016) 10(5):e0004715. doi:10.1371/journal.pntd.0004715

72. Habuchi S, Tsutsui H, Kochaniak AB, Miyawaki A, van Oijen AM. mKikGR, a monomeric photoswitchable fluorescent protein. PLoS One (2008) 3(12):e3944. doi:10.1371/journal.pone.0003944

73. Wilson J, Huynh C, Kennedy KA, Ward DM, Kaplan J, Aderem A, et al. Control of parasitophorous vacuole expansion by LYST/Beige restricts the intracellular growth of Leishmania amazonensis. PLoS Pathog (2008) 4(10):e1000179. doi:10.1371/journal.ppat.1000179

74. Real F, Mortara RA. The diverse and dynamic nature of Leishmania parasitophorous vacuoles studied by multidimensional imaging. PLoS Negl Trop Dis (2012) 6(2):e1518. doi:10.1371/journal.pntd.0001518

75. Kloehn J, Saunders EC, O'Callaghan S, Dagley MJ, McConville MJ. Characterization of metabolically quiescent Leishmania parasites in murine lesions using heavy water labeling. PLoS Pathog (2015) 11(2):e1004683. doi:10.1371/journal.ppat.1004683

76. Bruijnzeel PL, Uddin M, Koenderman L. Targeting neutrophilic inflammation in severe neutrophilic asthma: can we target the disease-relevant neutrophil phenotype? J Leukoc Biol (2015) 98(4):549-56. doi:10.1189/ jlb.3VMR1214-600RR

77. Carneiro MB, Hohman LS, Egen JG, Peters NC. Use of two-photon microscopy to study Leishmania major infection of the skin. Methods (2017) 127:45-52. doi:10.1016/j.ymeth.2017.04.012

78. Muller AJ, Aeschlimann S, Olekhnovitch R, Dacher M, Spath GF, Bousso P. Photoconvertible pathogen labeling reveals nitric oxide control of Leishmania major infection in vivo via dampening of parasite metabolism. Cell Host Microbe (2013) 14(4):460-7. doi:10.1016/j.chom.2013. 09.008

79. Pillay J, Kamp VM, van Hoffen E, Visser T, Tak T, Lammers JW, et al. A subset of neutrophils in human systemic inflammation inhibits $\mathrm{T}$ cell responses through Mac-1. J Clin Invest (2012) 122(1):327-36. doi:10.1172/ jci57990

80. Marini O, Costa S, Bevilacqua D, Calzetti F, Tamassia N, Spina C, et al. Mature CD10+ and immature CD10- neutrophils present in G-CSF-treated donors display opposite effects on T cells. Blood (2017) 129(10):1343-56. doi:10.1182/blood-2016-04-713206

81. Amulic B, Cazalet C, Hayes GL, Metzler KD, Zychlinsky A. Neutrophil function: from mechanisms to disease. Annu Rev Immunol (2012) 30:459-89. doi:10.1146/annurev-immunol-020711-074942

82. McCaffrey RL, Allen LA. Francisella tularensis LVS evades killing by human neutrophils via inhibition of the respiratory burst and phagosome escape. J Leukoc Biol (2006) 80(6):1224-30. doi:10.1189/jlb.0406287

83. Simons MP, Nauseef WM, Apicella MA. Interactions of Neisseria gonorrhoeae with adherent polymorphonuclear leukocytes. Infect Immun (2005) 73(4):1971-7. doi:10.1128/IAI.73.4.1971-1977.2005

84. van Zandbergen G, Gieffers J, Kothe H, Rupp J, Bollinger A, Aga E, et al. Chlamydia pneumoniae multiply in neutrophil granulocytes and delay their spontaneous apoptosis. J Immunol (2004) 172(3):1768-76. doi:10.4049/ jimmunol.172.3.1768

85. Spinner JL, Winfree S, Starr T, Shannon JG, Nair V, Steele-Mortimer O, et al. Yersinia pestis survival and replication within human neutrophil 
phagosomes and uptake of infected neutrophils by macrophages. J Leukoc Biol (2014) 95(3):389-98. doi:10.1189/jlb.1112551

Conflict of Interest Statement: The authors declare that the research was conducted in the absence of any commercial or financial relationships that could be construed as a potential conflict of interest.
Copyright $\odot 2017$ Regli, Passelli, Hurrell and Tacchini-Cottier. This is an open-access article distributed under the terms of the Creative Commons Attribution License (CC BY). The use, distribution or reproduction in other forums is permitted, provided the original author(s) or licensor are credited and that the original publication in this journal is cited, in accordance with accepted academic practice. No use, distribution or reproduction is permitted which does not comply with these terms. 